\title{
Characteristics of bamboo defects in peapod-grown double-walled carbon nanotubes
}

\author{
V. Zólyomi, ${ }^{1,2}$ J. Koltai, ${ }^{3}$ D. Visontai, ${ }^{1}$ L. Oroszlány, ${ }^{3}$ Á. Rusznyák, ${ }^{3}$ I. László, ${ }^{4}$ and J. Kürti ${ }^{3}$ \\ ${ }^{1}$ Physics Department, Lancaster University, LA1 $4 Y B$ Lancaster, United Kingdom \\ ${ }^{2}$ Research Institute for Solid State Physics and Optics, Hungarian Academy of Sciences, P.O. Box 49, H-1525 Budapest, Hungary \\ ${ }^{3}$ Institute of Physics, Loránd Eötvös University, Pázmány Péter sétány 1/A, H-1117 Budapest, Hungary \\ ${ }^{4}$ Department of Theoretical Physics, Budapest University of Technology and Economics, H-1111 Budafoki út. 8., Budapest, Hungary
}

(Received 2 July 2010; published 11 November 2010)

\begin{abstract}
Single-walled carbon nanotubes can function as nanoscale reaction chambers for growing smaller nanotubes within the host tube from encapsulated fullerenes by annealing. The diameter of the host outer tube restricts the diameter of the inner tube due to van der Waals interactions but not its chirality: it is possible that inner tubes with different chiralities start to grow in different places at the same time. A straight junction occurs at the connection of these two tubes which we refer to as bamboo defects. We show that localized states appear in the calculated density of states associated with these bamboo defects, some of them close to the Fermi level, and present a detailed theoretical study of ballistic transport through double-walled tubes where the inner shell contains bamboo defects. We find that the presence of bamboo defects should be possible to detect through electronic-transport measurements and the number of bamboo defects per unit length can be extracted from the structure of the resonances appearing in the transmission coefficient.
\end{abstract}

DOI: 10.1103/PhysRevB.82.195423

PACS number(s): 61.48.De, 71.15.Mb, 73.63.-b

\section{INTRODUCTION}

Peapods-single-walled carbon nanotubes filled with fullerenes - are of great interest in nanoscience due to their rich physics. ${ }^{1}$ One particular use of peapods is the synthesis of double-walled carbon nanotubes (DWCNTs): by annealing peapods the fullerenes within the host nanotube slowly coalesce and transform into an inner nanotube. ${ }^{2}$ The formation of the inner tubes has been confirmed by Raman spectroscopy. ${ }^{3,4}$ However, there is a major difference between such peapod-grown DWCNTs and other (e.g., chemical-vapor deposition grown) DWCNTs: NMR measurements on peapod-grown DWCNT samples have shown that the inner shells of such tubes exhibit a nearly uniformly metallic spin relaxation, ${ }^{5}$ suggesting that all inner tubes are metallic. This contradicts the fact that the chirality distribution of the inner tubes is only restricted by the diameter of the outer shell, and therefore contains semiconducting tubes such as $(6,4)$, as shown by Raman measurements. ${ }^{3}$ Furthermore, it has been shown that interwall interaction does not result in a semiconductor-metal transition for most inner tubes, ${ }^{6}$ hence the surprising result of the aforementioned NMR measurements is still unexplained. Recently, it was reported that the inner tubes exhibit an unexpectedly large electron spin resonance (ESR) signal, showing features reminiscent of localized states appearing during annealing. ${ }^{7}$ Furthermore, when grinding such samples the Raman signal of the inner tubes disappears suggesting that the inner tubes are not as durable as the outer shells, i.e., they break easily under mechanical strain. ${ }^{8}$

All of these experimentally observed features can be explained by the presence of a special kind of defect which could, in principle, appear in the inner walls during the growth process. For a given outer tube there are several different inner tubes which could grow during the annealing, since the van der Waals distance only restricts the diameter of the inner shell and not its chirality. ${ }^{3,4}$ At the very least, left- and right-handed varieties of the same chiral nanotube could grow in a given outer tube at the same time, e.g., $(6,4)$ and $(4,6)$. Furthermore, there is no reason why the inner shell could not start to grow in multiple places along the tube axis simultaneously. Therefore it is possible that during the annealing process the fullerenes start to coalesce at different places at the same time and inner tubes with different chiralities or with the same chirality but different handedness emerge. When these tubes of different chiralities come into contact, a junction will be formed. Such a junction is illustrated in Fig. 1, showing the case of the junction of a $(6,4)$ and a $(4,6)$ nanotube. In the remainder of the paper, we will refer to these junctions as bamboo defects. The presence of such defects would introduce mechanical strain at the junctions-especially when different chiralities are involved and the junction would prefer a bent geometry as opposed to the straight geometry enforced upon it by the outer shellwhich should account for the fragile nature of the inner shell and, as we will show below, localized defect states appear at the junctions which are very close to the Fermi level and

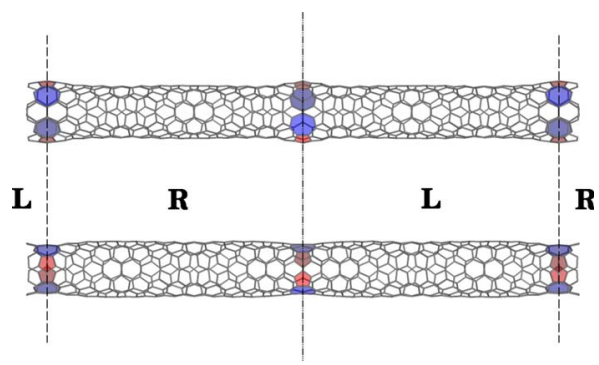

FIG. 1. (Color online) The geometry of the bamboo defect in a $(6,4)-(4,6)$ nanotube junction. The top and bottom panels are different views of the same geometry. Pentagons and heptagons are highlighted within the junction. The diameter of the tube expands near the pentagon defects and contracts near the heptagon defects. There are two nonequivalent junctions in the unit cell, the length of which depends on the density of junctions ( $65 \AA$ in this case). 
could, in principle, provide an explanation for the aforementioned NMR and ESR measurements.

In this paper we present a detailed study of the bamboo defects on the example of the $(6,4)$ inner tube. We employ first-principles density-functional theory (DFT) to obtain the optimal geometry and the electronic density of states. We use a tight-binding scheme to describe the electronic-transport properties of a double-walled tube comprising a $(10,10)$ outer tube and the aforementioned inner shell. We show that due to the presence of defect states localized on the bamboojunctions resonance dips appear in the transmission profile of a double-walled tube which are completely absent if there are no junctions, hence the presence of the bamboo defects can be confirmed through transport measurements. Furthermore, we show that the bamboo-defect density (i.e., the number of bamboo defects per unit length) can be determined from the resonance profile.

\section{METHOD}

The starting geometries of the bamboo junctions were constructed based on simple symmetry considerations which define the topology of the junction. ${ }^{9}$ We took two finite pieces of right-handed and left-handed nanotubes and connected them in a way that-beyond the usual hexagonsonly pentagons and heptagons appear in the carbon lattice, since these are the defects that emerge in Stone-Wales transformations. ${ }^{10}$ Once the topology was found, we performed a simple preoptimization by setting all bond lengths to the usual $1.42 \AA$ value and relaxing the structure with simple molecular dynamics. The resulting geometry was then used to construct the input for the DFT calculations (full geometry optimization and density of states, see below).

In order to perform calculations on realistic system sizes we need periodic boundary conditions. If we have a junction of a right-handed and a left-handed nanotube of the same chiral index, then the unit cell must contain two nonequivalent junctions if we are to apply periodic boundary conditions. This can be constructed as follows. Since there is a mirror plane symmetry at the junction (perpendicular to the tube axis), if we take an arbitrary finite piece of the nanotube-which contains exactly one bamboo junction and is long enough such that the geometry at one of the ends of this finite piece corresponds to that of a pristine nanotube and apply the $U$ symmetry operation ${ }^{11}$ to the system (i.e., a $180^{\circ}$ rotation about the symmetry axis perpendicular to the tube surface and going through the middle of a carboncarbon bond) at a point far enough from the junction then we will end up with a geometry which contains two nonequivalent junctions, one of which connects a left-handed piece to a right-handed one while the other connects the same righthanded piece to another left-handed one. By cutting this structure at the mirror planes of the two equivalent junctions we obtain the unit cell for the periodic calculation (see Fig. 1).

This geometry was optimized with first-principles density-functional theory; we used the Vienna $a b$ initio Simulation Package (VASP) (Refs. 12-14) in the local-density approximation (LDA) with the projector augmented-wave method. ${ }^{15,16}$ After obtaining the optimal geometry, we calculated the LDA density of states and used the junction geometry to construct longer supercells for the transport calculations (see below). During the LDA relaxation of the geometries we applied a plane-wave cut-off energy of 500 $\mathrm{eV}$. Having more than 300 atoms per unit cell, it proved to be enough to use a single $k$ point in this step. Optimization was performed until all forces fell below $3 \mathrm{meV} / \AA$. The electronic density of states was obtained from the band structure using a Green's function method; the band structure itself was calculated in a total of 11 irreducible $k$ points.

Once the geometry is fully optimized, we can extract the parameters of the screw-axis operation of the nanotube ${ }^{11}$ from the results. A nanotube can be built up by consecutively applying the screw-axis symmetry operation to the atoms of the helical unit cell (which contains $2 g c d$ atoms, where $g c d$ is the greatest common divisor of the chiral indices), i.e., the geometry is obtained by applying

$$
\left(C_{\alpha} \mid a\right)^{t}, \quad t=0, \pm 1, \pm 2, \ldots,
$$

where $C_{\alpha}$ is the rotation and $a$ is the translation component of the screw-axis operation, and $t$ is an integer. In a nanotube containing bamboo junctions the parameters $a$ and $\alpha$ vary along the length of the tube, as does the radial coordinate of the atoms. The size of a bamboo junction can be determined by looking at these parameters and calculating the distance measured from the middle of the junction where the length dependence falls off. Beyond this distance the helical symmetry could be used to elongate the structure by increasing the normal section of the tubes resulting in larger distances between neighboring bamboo defects. This method was applied to construct the inner shells of the double-walled nanotubes used as input for a series of calculations to determine the effect of bamboo defects on electron transport.

The electronic transport calculations were performed using the well-known Landauer-Büttiker formalism. ${ }^{17}$ The Hamiltonians used in the transport calculations were constructed using the intermolecular Hückel (IMH) model, which is a tight-binding model where a distance-dependent tight-binding scheme describes the interactions between different layers of a multiwalled nanotube while the physics of the individual layers is described by a nearest-neighbor tightbinding approximation. ${ }^{18,19}$ The IMH model is known to give a good description of intershell interactions in double-walled and multiwalled carbon nanotubes. $6,20,21$

\section{BAMBOO STATES}

The optimized geometry of the $(6,4)-(4,6)$ nanobamboo junction can be seen in Fig. 1. In this geometry the junction is straight and contains two heptagon-pentagon defects. The chosen unit cell contains 536 atoms and is $65.07 \AA$ long. There are two nonequivalent junctions in a single unit cell, so the distance between the junctions is about $32 \AA$.

Figure 2 shows the spatial dependence of the local tube radius and the helical translation parameter along the tube axis. The junction is on the left-hand side of the figure. All these parameters are oscillating around the value corresponding to the pristine $(6,4)$ nanotube and their difference is de- 


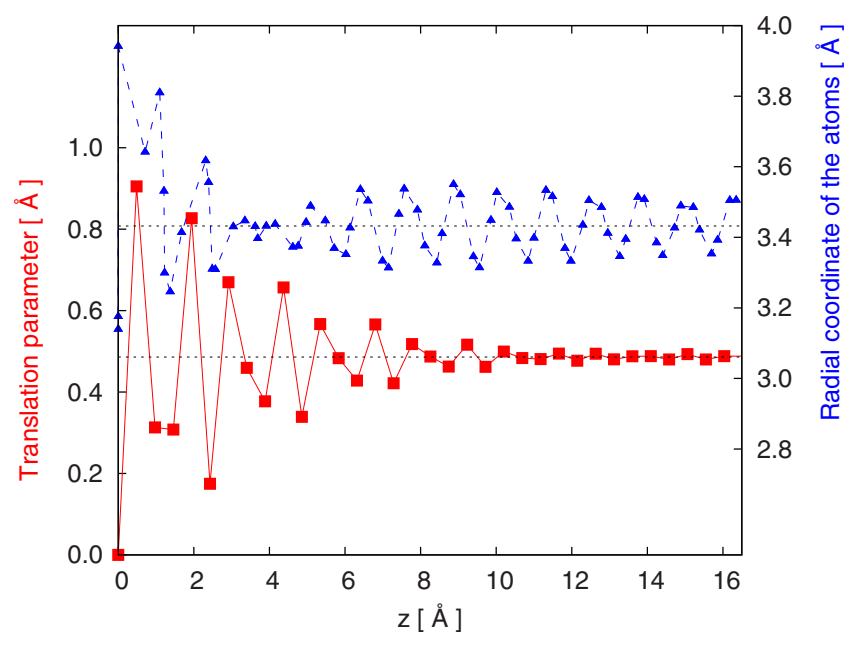

FIG. 2. (Color online) The helical translation parameter of the screw-axis symmetry operation and the radial coordinate of the atoms of the $(6,4)$ nanotube in a $(6,4)-(4,6)$ nanobamboo geometry. The origin of the $z$ axis is placed at one of the junctions, and the figure is plotted up to half way toward the next junction. The parameters converge toward the values of the pristine nanotube as we move away from the junction. The rotation parameter of the screw axis (not shown) is more-less constant and corresponds to the pristine value.

creasing with the distance from the junction. Although the radius is still somewhat oscillating around the pristine value far away from the junction, the wild oscillation is already flattened out at $4 \AA$. Based on these parameters, the size of a bamboo defect can be estimated to be around a maximum of two times $8-10 \AA$. This confirms that our unit cell is large enough that the interaction between the two junctions is negligible. Therefore it is possible to build longer structures by simply extending the nanotube based on the averaged geometrical parameters half way between the junctions, and there is no need for any further geometry optimization. However, if we want to decrease the distance between the junctions we always have to perform a full geometry optimization at the DFT level.

We also calculated the electronic band structure and the density of states for this structure at the DFT level. The density of states (DOS) is plotted in Fig. 3. For comparison the DOS of the pristine $(6,4)$ was also calculated. (The DOS was obtained from the band structure using the well-known formula $\operatorname{DOS}(E)=\frac{2}{\pi N_{a t}} \operatorname{Im} \Sigma_{b} \int \frac{1}{E-E_{b}(k)-i \gamma} d k$, where $N_{a t}$ is the number of atoms, $E_{b}(k)$ is the $b$ th energy band, the sum goes over all bands of the system, the integral goes over the entire Brillouin zone, and $\gamma$ is a broadening parameter which in our calculation was $0.02 \mathrm{eV}$.) The most eye-catching difference between the two cases is the pair of sharp peaks near the Fermi energy. In the band structure these two peaks correspond to two almost entirely flat bands, suggesting that these bands originate from localized states. This is confirmed by tight-binding calculations as well, as we discuss below.

One of the most interesting features of these defect states is that their energy strongly depends on the distance of the bamboo defects. By varying the distance between the junctions the position of these peaks could be tuned such that,

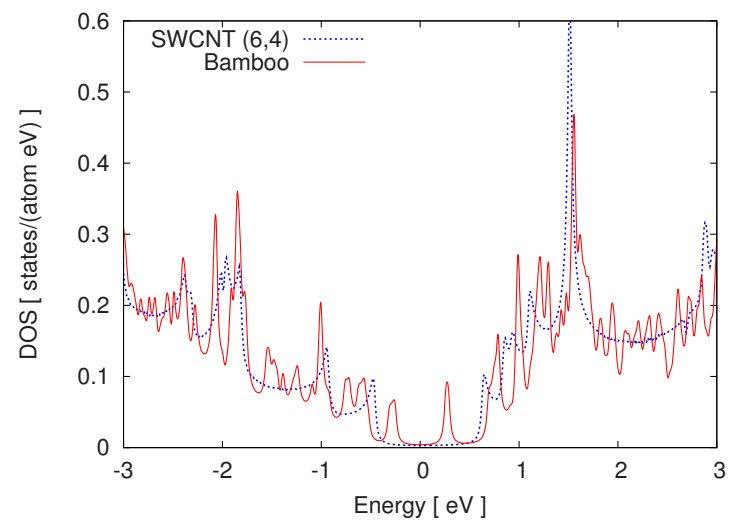

FIG. 3. (Color online) LDA electronic density of states for the $(6,4)-(4,6)$ nanobamboo geometry (solid) with $32.54 \AA$ between neighboring junctions compared with the density of states of the pristine $(6,4)$ nanotube (dashed).

e.g., at a length of only $6.26 \AA$ between two junctions, the peaks were tuned exactly to the Fermi energy (see Fig. 4). On the other hand at such a high defect density the DOS has completely forgotten the structure of the original $(6,4)$ nanotube, all the Van Hove singularities have disappeared. However, this is not supported by Raman measurements on DWCNTs grown from peapods which clearly show that there is no splitting for the $E_{i i}$ optical transitions of the inner tubes, confirming the presence of Van Hove singularities in the DOS of the inner tubes. ${ }^{4}$ This allows us to give an upper estimate of the bamboo-defect density by calculating the DOS with different defect distances, gradually increasing the distance of the defects until the Van Hove peaks characteristic of a pristine nanotube emerge. This calculation involves geometries containing up to 7000 atoms, hence we used a simple nearest-neighbor tight-binding approximation with a hopping energy of $2.5 \mathrm{eV}$ for this step (i.e., the intramolecular part of the IMH model). We have found that the Van Hove singularities in the tight-binding level DOS of the pristine $(6,4)$ nanotube are restored in the bamboo-junction calculation if the distance between the bamboo defects is around $300 \AA$ or larger. This gives us a lower estimate of

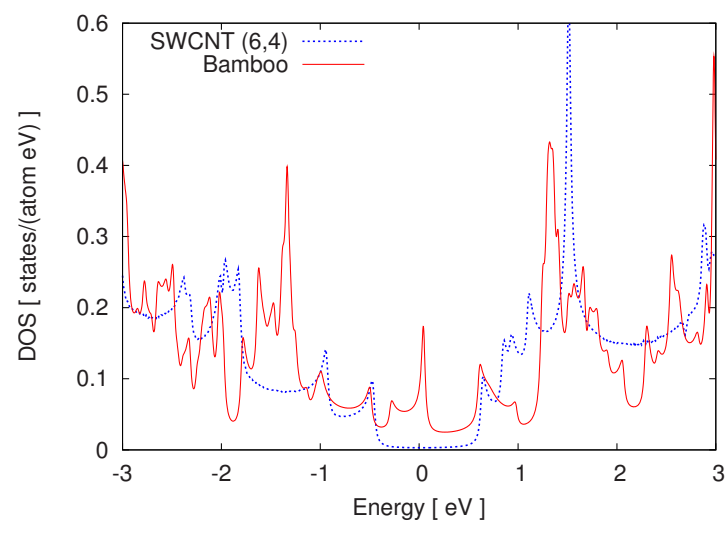

FIG. 4. (Color online) LDA electronic density of states for the $(6,4)-(4,6)$ nanobamboo geometry (solid) with $6.26 \AA$ between neighboring junctions compared with the density of states of the pristine $(6,4)$ nanotube (dashed). 

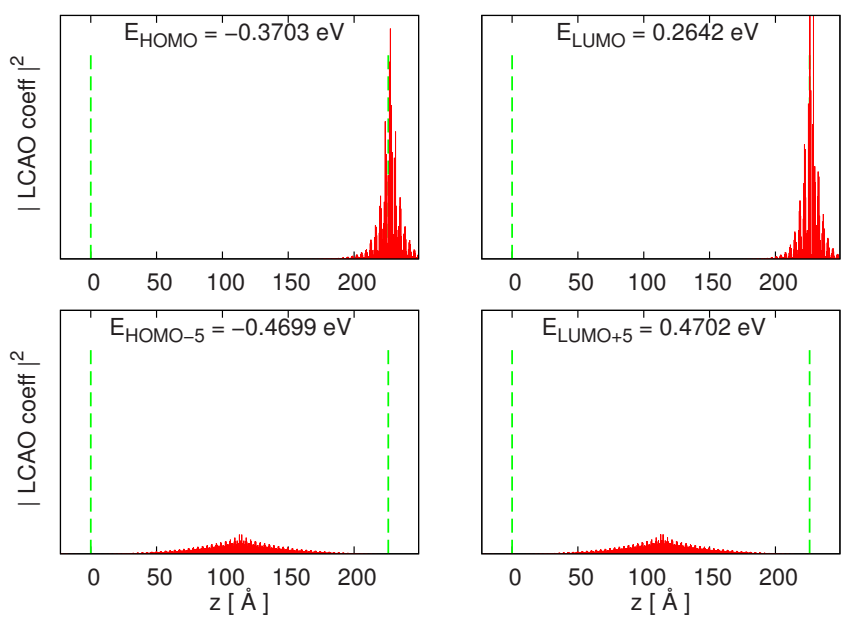

FIG. 5. (Color online) The square of the magnitude of the LCAO coefficients of the HOMO, LUMO, HOMO-5, and LUMO +5 electron states in the first half of the $(6,4)-(4,6)$ nanobamboo unit cell (i.e., the LCAO coefficients are the coefficients $c_{i}$ obtained from the Hückel calculations, $\hat{H} \underline{c}_{i}=E_{i} \hat{S}_{c_{i}}$, where $\hat{H}$ and $\hat{S}$ are the Hamiltonian and overlap matrices, respectively, and $\underline{c}_{i}$ is the $i$ th solution of the eigenvalue equation with eigenvalue $E_{i}$, with each component of the vector $\underline{c}_{i}$ corresponding to one of the atoms in the nanotube). The states closest to the Fermi level are strongly localized while the states far from the Fermi level are delocalized as expected.

$300 \AA$ for the average distance of the junctions, i.e., an upper estimate of one junction per $300 \AA$ for the bamboodefect density. Note that in an actual experiment the distance between neighboring junctions is most likely not a constant value, rather, it has a distribution such that some junctions are very close while others are very far from one another. This means that localized states of different energies can appear within the band gap, which may form a band of their own where low-energy excitations should be possible. This may explain the NMR and ESR measurements mentioned in Sec. I, as we discuss below.

Beyond being able to estimate the upper bound of the bamboo-defect density, the tight-binding calculation is also helpful for confirming that the sharp peaks near the Fermi level are indeed localized states. The DOS near the Fermi level is very similar on the tight-binding and DFT levels (in particular, the energy of the localized peaks is nearly identical in the two calculations), hence the tight-binding linear combination of atomic orbitals (LCAO) coefficients of the electron states corresponding to the defect peaks in the DOS can be used to easily determine whether the defect states are localized or not. Figure 5 shows the LCAO coefficients for four of the tight-binding electron states. In this figure, the $z$ coordinate is along the tube axis and there are two junctions, their locations are marked by the dashed lines. The upper panel depicts the highest occupied molecular orbital (HOMO) and lowest unoccupied molecular orbital (LUMO). These states are localized on the bamboo junction at the right edge of the figure. Each of these states is doubly degenerate and their pairs are localized on the other junction. The reason for this degeneracy is simply the inversion symmetry of the geometry. The lower panel depicts two states that are far from the Fermi level, HOMO-5 and LUMO+5. Both of these states are highly delocalized and the vast majority of the electron states are like this. Thus we can conclude that the bamboo defects are characterized by the appearance of localized states near the Fermi energy.

\section{SIGNIFICANCE}

The presence of localized states in the DOS originating from the junctions studied here has a significant impact on the physical properties of the inner tubes. As mentioned in Sec. I, recent measurements have shown that the ESR signal of the inner shells of peapod-grown DWCNTs is unusually high, and localized states may be responsible for this phenomenon. ${ }^{7}$ Furthermore, NMR spin-relaxation measurements suggest that all inner tubes are metallic or at least they all have states near the Fermi level. ${ }^{5}$ The presence of bamboo junctions could explain this finding for the following reasons.

As detailed above, there is a lower bound for the average distance of the bamboo junctions based on the features of the Raman measurements. However, this is only the average, in a real experiment these distances likely have some kind of distribution: some junctions are very close others are very far apart. Therefore, since the energy of the bamboo states depends on the distance of bamboo junctions, the energy of the bamboo states will also have a distribution, resulting in the appearance of a series of localized states in the band gap with different energies. These states together form a defect band which is very close to the Fermi level. Within this band, low-energy excitations may be possible, although selection rules associated with the localized nature of the states within the band may prohibit them from participating in the NMR relaxations.

However, in a real experiment the tubes on the two sides of the bamboo junctions should have different chiralities most of the time, which have different Fermi levels. Therefore, if tubes of different chiralities are connected by a bamboo defect, it may be possible for one nanotube to dope the other. For example, there is a chance that when a semiconductor and a metal connect, charge transfer takes place between the bulk bands of the semiconductor nanotube and the localized states of the junction, resulting in the appearance of low-energy excitations in the band structure of the semiconducting tube after this shift in occupations takes place. This is of course an effect which requires detailed studies, and at this stage we cannot say whether the energetics in such a system would allow for such charge redistribution. Furthermore, due to the shape of the junction, interactions with the outer tube should be considered when attempting to look at this effect. In fact, it is already an important question whether the bond lengths and bond angles in the junction will be significantly modified by the presence of the outer tube as compared to a single-walled scenario used in our calculations.

Beyond the aforementioned measurements, the bamboo junctions are very important for the structural stability of the nanotubes as well. Single-walled tubes are known to have very high stability toward mechanical manipulations due to 
their high Young's moduli. The inner tubes of peapod-grown double-walled tubes are, however, known to be mechanically unstable, they are destroyed by grinding the sample while the outer tubes remain intact. ${ }^{8}$ This effect can be explained by the presence of the junctions discussed here. Each junction is formed at the cost of the appearance of a number of pentagon-heptagon defects, which make the junctions rigid and therefore they should be more likely to break than a pristine nanotube. Furthermore, when two different chiralities merge, the junction is typically not straight and being forced into a straight outer shell, even if the outer tube bends to accommodate the inner tube, strain will arise associated with the external boundary condition, which will further contribute to possible breaking of the junctions if the sample is subjected to external mechanical strain. The shorter the normal pieces between consecutive junctions the more fragile the inner tubes should be. Thus the presence of these junctions could easily explain why the inner tubes seem to break when grinding the sample.

\section{ELECTRONIC TRANSPORT}

So far we have demonstrated the basic properties and the significance of bamboo defects in the inner walls of peapodgrown DWCNTs. However, none of the above cited experiments give any conclusive confirmation of the existence of these bamboo junctions. One possible way of identifying bamboo defects in a nanotube sample could be the use of high-resolution transmission electron microscopy along the full length of a series of peapod-grown DWCNTs, which is an experiment that would be most worthwhile to perform. Another possibility lies in the study of the electronictransport properties of DWCNTs. In the following, we will demonstrate that the presence of bamboo junctions in a DWCNT can be detected through transport measurements. Furthermore, we will show that the bamboo-defect density along the nanotube can be extracted from the resonance profile in the electronic transmission.

Metallic carbon nanotubes are ballistic conductors with a widely studied transmission profile. The number of open channels in an armchair nanotube is 2 near the Fermi energy ${ }^{11}$ and shows a stepwise increase as we move away from the Fermi level. Like in the case of any molecular nanowire, interaction with molecules attached to the nanotube will produce resonance dips in the transmission coefficient: the transmission probability suffers a significant decrease at the energies corresponding to the energy levels of the attached molecule. Similarly, if molecules are encapsulated within a nanotube, the interaction causes the transmission to decrease at certain energies. In the following we will show that in DWCNTs, where the electron transport is through the outer layer, the presence of bamboo defects in the inner shell produces signature resonances in the transmission probability which are absent if the inner shell is a pristine nanotube.

We have performed calculations on the electronictransport properties of DWCNTs comprising a $(10,10)$ outer shell and a $(6,4)$ inner shell. We considered a two-contact scenario where the leads are attached to the outer shell of the

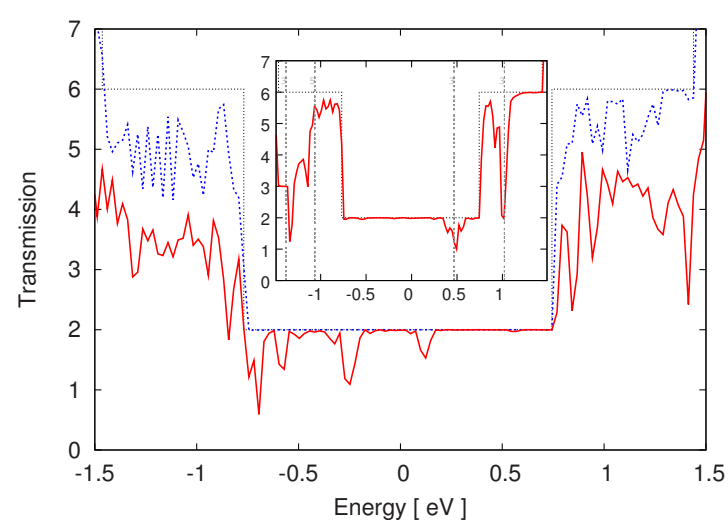

FIG. 6. (Color online) Transmission probability of the $(6,4) @(10,10)$ DWCNT obtained with the IMH model within the Landauer-Büttiker formalism. The dotted (black) line shows the number of open channels through the $(10,10)$ nanotube while the solid (red) and dashed (blue) curves correspond to the transmission of the DWCNT when the inner shell is a $(6,4)-(4,6)$ nanobamboo and a pristine $(6,4)$ nanotube, respectively. The inset shows the transmission through a fullerene-nanotube peapod $\left[\mathrm{C}_{60}\right.$ molecules encapsulated in a $(10,10)$ nanotube].

DWCNT. The outer shell is considered infinite while the inner shell is finite; only the outer shell is in contact with the leads. If there was no inner shell, the transmission probability from one end of the $(10,10)$ nanotube to the other would be a step function, as determined by the number of open channels. This is shown as the dotted line in Fig. 6. However, in the case of a DWCNT, interaction between the two shells reduces the transmission probability. The transmission of a $(6,4) @(10,10)$ DWCNT (with contacts touching only the outer shell) is shown as the dashed line in Fig. 6. As it can be seen, the transmission is decreased at energies that are more than roughly $0.8 \mathrm{eV}$ from the Fermi level, as expected. There is no change at smaller energies since the inner tube is a $(6,4)$ nanotube, which is a semiconductor and has no states in that energy region. This was the case of a pristine inner tube.

Now, if we perform the same calculation with a $(6,4)$ $(4,6)$ nanobamboo geometry, then due to the localized states near the Fermi level, we would expect some kind of change in the transmission through the outer shell at low energies as well. Indeed, as it can be seen in Fig. 6, several dips appear between -0.8 and $0.3 \mathrm{eV}$ for the bamboo-junction geometry (solid line). This effect is shown in greater detail in Fig. 7, which zooms in on the low-energy region. The figure clearly shows that the features in the bamboo-junction geometry are completely absent if the inner tube is a pristine nanotube. Furthermore, there are multiple peaks appearing in the transmission profile and the peak structure depends on the average distance of the junctions, this is discussed below.

For comparison, we have also calculated the transmission for a peapod geometry, replacing the inner tube with $\mathrm{C}_{60}$ fullerene molecules. The result of this calculation is shown in the inset of Fig. 6. As it can be seen, there are some features in the transmission profile due to the interaction between the fullerenes and the nanotube, as expected. These features 


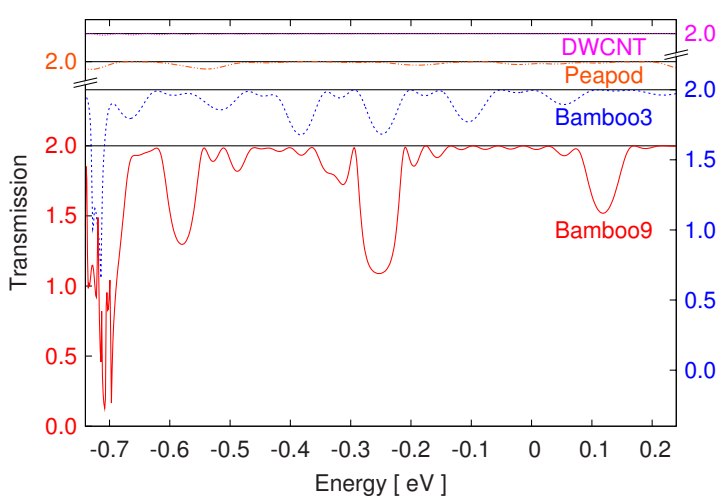

FIG. 7. (Color online) Low-energy region of the transmission probability of the $(6,4) @(10,10)$ DWCNT. The curve labeled DWCNT depicts the case when the inner shell is a pristine $(6,4)$ while the ones labeled Bamboo3 and Bamboo9 depict the cases when the inner shell is a $(6,4)-(4,6)$ nanobamboo geometry. The Bamboo3 geometry contains 3 junctions which are roughly $80 \AA$ apart, while the Bamboo9 geometry contains nine junctions which are roughly $30 \AA$ apart. (In both cases full periodic boundary conditions were applied to the outer shell geometry which was of length $340 \AA$ A.) The curve labeled peapod depicts the transmission through a peapod geometry. The four curves are shifted along the transmission axis for sake of clarity. For the top two curves, only the maximum transmission (2.0) is printed on the axes with the 2.0 on the left corresponding to the peapod curve and the 2.0 on the right corresponding to the DWCNT curve. For the bottom two curves, the scale on the left side corresponds to the Bamboo9 curve while the scale on the right side corresponds to the Bamboo 3 curve.

appear at energies corresponding to the energy levels of $\mathrm{C}_{60}$, which are marked by vertical lines. These features, however, are far from the Fermi level and do not overlap with the peaks originating from the bamboo defects, as it can be clearly seen in Fig. 7. These results show that neither the pristine nanotube nor any residual $\mathrm{C}_{60}$ molecules produce features that overlap with those originating from the bamboo defects. Therefore, if such peaks are found at low energies in a transport measurement on peapod-grown DWCNTs that have a semiconducting inner shell, they are a clear indication of the presence of bamboo junctions in the inner wall.

As noted above, the bamboo junctions cause multiple dips to appear in the transmission probability. Furthermore, as it can be seen in Fig. 7, the fine structure of these dips depends on the distance of the junctions. This fine structure can be used to determine the average distance of the junctions, which is a characteristic length scale for these structures.

In Fig. 7, we have shown results for two different bamboo-junction distances, spacings of roughly 30 and $80 \AA$. In both cases there are a number of easily identifiable dips where the transmission decreases significantly. The energy spacing of these dips is larger when the spacing of the junctions is smaller. In fact, the distance of these main dips in energy is inversely proportional to the distance of the junctions in real space. The reason for this is simple. Since the energy spectrum of the host nanotube is linear in this region, spacing in energy is directly proportional to a spacing in $k$ space, which is, of course, inversely proportional to a spacing in real space. In other words, due to the linear energy dispersion of carbon nanotubes at low energies, the separation of the strong reflection energies is inversely proportional to the distance of the junctions. This should be visible in the transmission even if the junctions are not spaced evenly, therefore it should be possible to extract the average junction distance from the energy dependence of the transmission coefficient.

Note that there are several smaller satellite peaks between the aforementioned strong reflection peaks in Fig. 7. These are artifacts of our calculation which only arise if the structure is fully periodic. They can be described by the KronigPenney model, ${ }^{22}$ and the number of satellite peaks is equal to $n-2$ for $n$ bamboo junctions. However, since in a real experiment the junctions are not evenly spaced and there should be nanotubes of different chiralities on the two sides of the junction in most cases, this feature will most certainly be averaged out. Only the main peaks should persist, which can be used to determine the average bamboo-defect density in the sample as detailed above.

It must be noted that in a real experiment the nanotubes contain traditional disorder in the form of, e.g., vacancies and Stone-Wales defects. In principal it could be possible that such defects will cause similar effects on the transmission as the bamboo junction and therefore the two cannot be distinguished. However, we argue that the bamboo junction is a unique type of defect that should produce a sharper signal than traditional defects due to the fact that, e.g., vacancies and Stone-Wales pairs are small defects involving a small number of atoms while a bamboo junction is an extended defect formed of several tens of atoms. While the bamboo junction is well localized along the tube axis, it extends around the full circumference of the nanotube, which is fundamentally different from the much more localized nature of the traditional defects which are much smaller. In the previous paragraph we argued that even when the bamboo junctions are randomly spaced as they likely are in a real sample, only the fine structure of the dips in the transmission will be averaged out and the main features will be retained. The same will not be true for, e.g., a Stone-Wales defect. Still, it might be possible for dips in the transmission caused by traditional defects to overlap the features caused by the bamboo junction, making it impossible to detect the aforementioned resonance dips. To test this we have performed a calculation on the nine-junction Bamboo9 geometry (see Fig. 7) with additional disorder taken into account. We have introduced nine Stone-Wales pairs into the Bamboo9 geometry scattered randomly along the length of the inner tube. We have calculated the transmission through this DWCNT and averaged over ten random distributions. Our results are depicted in Fig. 8. It can be seen that one of the main dips of the bamboo junction are indeed overlapped by a quite noisy signal produced by the newly introduced Stone-Wales pairs, making it impossible to detect this resonance dip. However, the other two main dips are still very clearly visible even in the presence of Stone-Wales pairs. Therefore we conclude that the bamboo junctions we propose should be detectable experimentally even in a real, disordered DWCNT. 


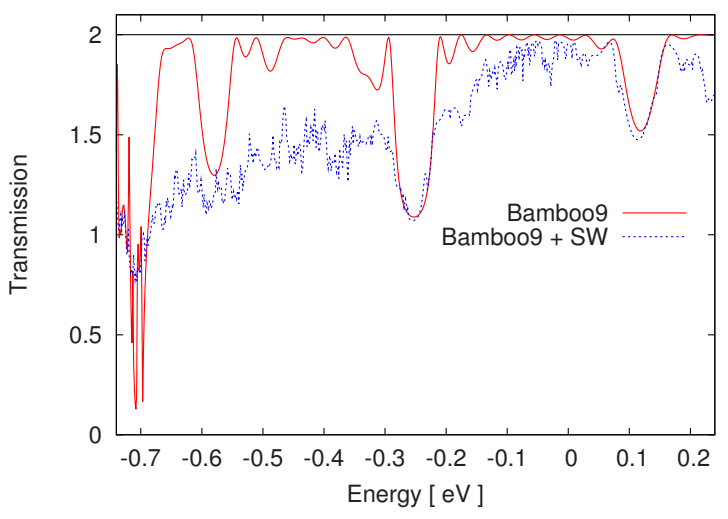

FIG. 8. (Color online) The effect of traditional disorder on the transmission probability of the $(6,4) @(10,10)$ nanobamboo DWCNT. The curve labeled Bamboo9 depicts the case when the inner shell is a $(6,4)-(4,6)$ nanobamboo geometry containing nine bamboo junctions which are roughly $30 \AA$ apart. The curve labeled Bamboo9+SW depicts the case when nine additional Stone-Wales pair defects are added to the previously mentioned geometry, randomly distributed on the inner tube. The latter curve was calculated after averaging over ten different random distributions of the StoneWales pairs. It can be seen that three of the two major features of the bamboo junction are still visible even if traditional disorder is taken into account, suggesting that the effect should be visible in a transport measurement on a real sample that contains traditional lattice defects.

\section{STABILITY}

Finally, we must address the question of the thermal stability of the bamboo junctions. In principle, one might think that at high temperatures the junctions could move along the axis of the nanotube similar to the movement of solitons. If this was the case, then the junctions could disappear during the annealing process since they could either move to the end of the nanotube where they could easily reconstruct (especially at an open end) or it would be possible for two junctions to cancel each other out in certain cases, similar to the annihilation of a soliton-antisoliton pair. For example, in our $(6,4)-(4,6)$ nanobamboo geometry, there are junctions that connect a left-handed piece to a right-handed one, and junctions that connect a right-handed one to a left-handed one. The former and latter junctions are mirror opposites of each other and, should they be mobile along the tube, they should be able to consume each other through Stone-Wales transformations. If, however, the bamboo defects are not mobile along the tube, then such cancellation cannot take place and the junctions will be stable toward high temperatures and should persist during the annealing.

We have performed molecular-dynamics simulations to address this question. We used the DL_POLY moleculardynamics package ${ }^{23}$ with the Tersoff potential ${ }^{24}$ and the NVT Berendsen thermostat. ${ }^{25}$ We ran the simulations for up to 3 ns in $0.5 \mathrm{fs}$ time steps. We used a double-walled geometry with a $(10,10)$ outer shell and a $(6,4)-(4,6)$ nanobamboo inner shell with eight bamboo junctions. All atoms were allowed to move. We have found that the bamboo defects are stable up to simulation temperatures as high as $3500 \mathrm{~K}$ (see Fig. 9). For comparison, previous calculations in the litera-

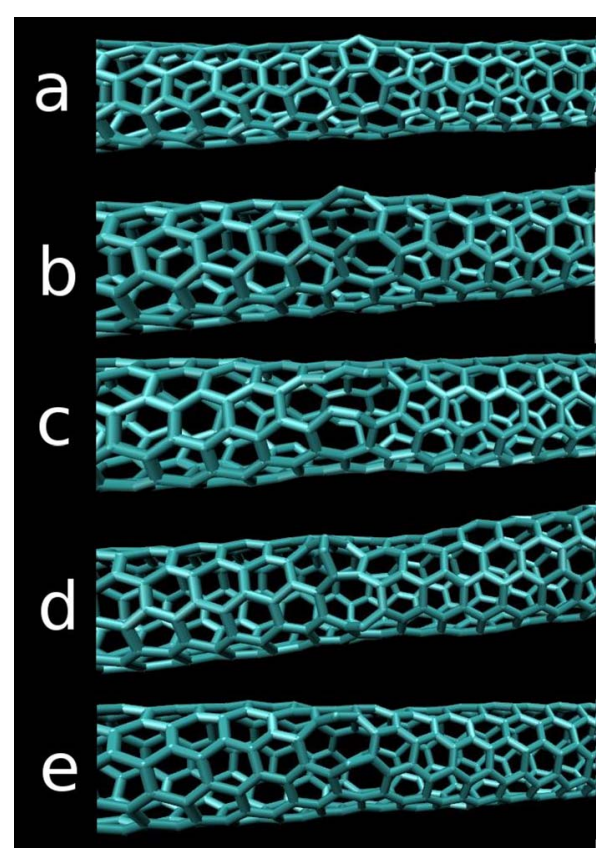

FIG. 9. (Color online) Structure of the inner shell of a $(6,4) @(10,10)$ DWCNT with eight bamboo junctions in the inner shell during a molecular-dynamics simulation at $3500 \mathrm{~K}$ using the Tersoff potential and the NVT Berendsen thermostat. Geometries taken at $0 \mathrm{~ns}, 0.01 \mathrm{~ns}, 0.2 \mathrm{~ns}, 0.5 \mathrm{~ns}$, and $3 \mathrm{~ns}$ from top to bottom, respectively.

ture with this method on peapods have shown that the method can describe the coalescence of $\mathrm{C}_{60}$ fullerenes, ${ }^{26}$ albeit the method predicts a much higher temperature for the coalescence than the experiments: the simulation predicts that coalescence begins at around $2500 \mathrm{~K}$ while the experiments take place at around $900 \mathrm{~K}$. Nevertheless, since our calculations show that the bamboo junction is stable at temperatures much higher than the $\mathrm{C}_{60}$ coalescence temperature predicted by the same method, we can safely conclude that the bamboo junctions are stable in the temperature range of the annealing, and therefore they should persist during the formation of the inner shells.

\section{CONCLUSION}

We presented a study of bamboolike junctions in the inner tubes of peapod-grown double-walled carbon nanotubes. We have demonstrated that the junctions are energetically stable and are likely to form during the synthesis of the inner tubes. Molecular-dynamics simulations have shown that the junctions are stable even at high temperatures. We found tunable localized states around the Fermi energy both with densityfunctional theory and tight-binding level calculations. These states were confirmed to be localized around the junctions by looking at the LCAO coefficients of the molecular orbitals. We have shown that the bamboo defects in the inner tube of a double-walled tube leave signature resonances in the electronic-transmission coefficient, thus the junctions should be possible to detect by transport measurements. Further- 
more, the average distance of neighboring bamboo defects can be extracted from the transmission resonance profile.

\section{ACKNOWLEDGMENTS}

Support from OTKA in Hungary (Grants No. F68852, No.
K60576, and No. K81492) and the EPSRC in the U.K. is gratefully acknowledged. V.Z. also acknowledges the Marie Curie IEF project NANOTRAN (Grant No. PIEF-GA-2008220094). We thank C. J. Lambert and V. I. Fal'ko for valuable discussions and F. Simon for providing motivation.
${ }^{1}$ B. W. Smith, M. Monthioux, and D. Luzzi, Nature (London) 396, 323 (1998).

${ }^{2}$ S. Bandow, M. Takizawa, K. Hirahara, M. Yudasaka, and S. Iijima, Chem. Phys. Lett. 337, 48 (2001).

${ }^{3}$ R. Pfeiffer, H. Kuzmany, C. Kramberger, C. Schaman, T. Pichler, H. Kataura, Y. Achiba, J. Kürti, and V. Zólyomi, Phys. Rev. Lett. 90, 225501 (2003).

${ }^{4}$ R. Pfeiffer, F. Simon, H. Kuzmany, and V. N. Popov, Phys. Rev. B 72, 161404 (2005).

${ }^{5}$ P. M. Singer, P. Wzietek, H. Alloul, F. Simon, and H. Kuzmany, Phys. Rev. Lett. 95, 236403 (2005).

${ }^{6}$ V. Zólyomi, J. Koltai, Á. Rusznyák, J. Kürti, Á. Gali, F. Simon, H. Kuzmany, Á. Szabados, and P. R. Surján, Phys. Rev. B 77, 245403 (2008).

${ }^{7}$ B. Náfrádi, N. M. Nemes, T. Fehér, L. Forró, Y. Kim, J. E. Fischer, D. E. Luzzi, F. Simon, and H. Kuzmany, Phys. Status Solidi B 243, 3106 (2006).

${ }^{8}$ F. Simon (unpublished).

${ }^{9}$ I. László, Croat. Chem. Acta 81, 267 (2008).

${ }^{10}$ A. J. Stone and D. J. Wales, Chem. Phys. Lett. 128, 501 (1986).

${ }^{11} \mathrm{~S}$. Reich, C. Thomsen, and J. Maultzsch, Carbon Nanotubes (Wiley-VCH Verlag GmbH, Berlin, 2004).

${ }^{12}$ G. Kresse and J. Hafner, Phys. Rev. B 48, 13115 (1993).
${ }^{13}$ G. Kresse and J. Furthmüller, Comput. Mater. Sci. 6, 15 (1996).

${ }^{14}$ G. Kresse and J. Furthmüller, Phys. Rev. B 54, 11169 (1996).

${ }^{15}$ P. E. Blöchl, Phys. Rev. B 50, 17953 (1994).

${ }^{16}$ G. Kresse and D. Joubert, Phys. Rev. B 59, 1758 (1999).

${ }^{17}$ M. Büttiker, Y. Imry, R. Landauer, and S. Pinhas, Phys. Rev. B 31, 6207 (1985).

${ }^{18}$ A. Lázár, P. R. Surján, M. Paulsson, and S. Stafström, Int. J. Quantum Chem. 84, 216 (2001).

${ }^{19}$ P. R. Surján, A. Lázár, and Á. Szabados, Phys. Rev. A 68, 062503 (2003).

${ }^{20}$ H. Shiozawa et al., Phys. Rev. B 77, 153402 (2008).

${ }^{21}$ Á. Szabados, L. P. Biró, and P. R. Surján, Phys. Rev. B 73, 195404 (2006).

${ }^{22}$ D. W. L. Sprung, H. Wu, and J. Martorell, Am. J. Phys. 61, 1118 (1993).

${ }^{23}$ W. Smith, I. T. Todorov, and M. Leslie, Z. Kristallogr. 220, 563 (2005).

${ }^{24}$ J. Tersoff, Phys. Rev. B 37, 6991 (1988).

${ }^{25}$ H. J. C. Berendsen, J. P. M. Postma, W. F. V. Gunsteren, A. Dinola, and J. R. Haak, J. Chem. Phys. 81, 3684 (1984).

${ }^{26}$ E. Hernández, V. Meunier, B. W. Smith, R. Rurali, H. Terrones, M. Buongiorno Nardelli, M. Terrones, D. E. Luzzi, and J.-C. Charlier, Nano Lett. 3, 1037 (2003). 